\title{
Magnetoresistance oscillations in superconducting strips: A Ginzburg-Landau study
}

\author{
G. R. Berdiyorov, ${ }^{1}$ X. H. Chao, ${ }^{2}$ F. M. Peeters,,${ }^{1, *}$ H. B. Wang, ${ }^{3}$ V. V. Moshchalkov, ${ }^{4}$ and B. Y. Zhu ${ }^{2}$ \\ ${ }^{1}$ Departement Fysica, Universiteit Antwerpen, Groenenborgerlaan 171, B-2020 Antwerpen, Belgium \\ ${ }^{2}$ National Laboratory for Superconductivity, Institute of Physics, and Beijing National Laboratory for Condensed Matter Physics, \\ Chinese Academy of Sciences, Beijing 100190, China \\ ${ }^{3}$ National Institute for Materials Science, Tsukuba 3050047, Japan \\ ${ }^{4}$ INPAC - Institute for Nanoscale Physics and Chemistry, Katholieke Universiteit Leuven, Celestijnenlaan 200D, B-3001 Leuven, Belgium
}

(Received 16 September 2012; revised manuscript received 19 November 2012; published 10 December 2012)

\begin{abstract}
Within the time-dependent Ginzburg-Landau theory we study the dynamic properties of current-carrying superconducting strips in the presence of a perpendicular magnetic field. We found pronounced voltage peaks as a function of the magnetic field, the amplitude of which depends both on sample dimensions and external parameters. These voltage oscillations are a consequence of moving vortices, which undergo alternating static and dynamic phases. At higher fields or for high currents, the continuous motion of vortices is responsible for the monotonic background on which the resistance oscillations due to the entry of additional vortices are superimposed. Mechanisms for such vortex-assisted resistance oscillations are discussed. Qualitative changes in the magnetoresistance curves are observed in the presence of random defects, which affect the dynamics of vortices in the system.
\end{abstract}

DOI: 10.1103/PhysRevB.86.224504

PACS number(s): 74.78.Na, 73.23.-b

\section{INTRODUCTION}

Low-dimensional superconducting systems, such as nanowires and nanoribbons, have recently received a revival of interest due to their unusual properties, which are absent in bulk superconductors. An example is the considerable enhancement of the critical parameters ${ }^{1-5}$ due to condensate confinement $^{6-10}$ and quantum-size oscillations. ${ }^{1}$ Quantumsize effects also modify the characteristic length scales, leading to the formation of novel Andreev-type states. ${ }^{11}$ For practical implementation of these systems, the intrinsic thermal ${ }^{12}$ and quantum ${ }^{13}$ fluctuations become very important, as they bring the system into the resistive state well below the superconducting transition temperature. Such fluctuations also result in oscillatory behavior of the resistance as a function of applied magnetic field in superconducting nanowires (see, e.g., Ref. 14), which are also very sensitive to imperfections in the system and the shape of the samples.

Magnetoresistance oscillations have also been observed in samples with transverse dimensions larger than the superconducting coherence length $\xi .{ }^{15,16}$ One possible explanation for such resistance oscillations is the formation of effective multiconnected (ringlike) structures ${ }^{17}$ (due to, e.g., the nonuniform thickness of the sample), which may lead to resistance oscillations similar to Little-Parks oscillations. ${ }^{18}$ However, nonperiodic behavior of the resistance oscillations is usually observed in experiments. ${ }^{15,16}$ Similar nonmonotonic behavior of the resistance is also a common feature of granular superconductors ${ }^{19}$ originating from the screening currents around phase-coherent loops of weakly linked superconducting grains. Such weak links can result in superconductinginsulator transition $\mathrm{s}^{20}$ and consequently in large magnetoresistance oscillations in a broad range of temperatures and magnetic fields. ${ }^{21}$ However, magnetoresistance anomalies have also been reported in well-prepared, low- $T_{c}$ superconductors, ${ }^{22}$ where the formation of such weak links is less favorable, in part due to the larger $\xi$. In spite of the increased number of theoretical and experimental works, the magnetoresistance oscillations in such samples are not well understood. ${ }^{16}$
In their original paper, Anderson and Dayem ${ }^{23}$ proposed an alternative explanation in which they assumed that the resistance peaks are due to dissipation resulting from the motion of vortices across the strip. Here, we present results of numerical simulations, which also reveal that the magnetoresistance oscillations originate from moving vortices. These vortices undergo different static and dynamic phases, because of the modification of the surface barrier for their entry and exit, due to competing interactions between the applied current and the Meissner currents. In addition, we found that the monotonic background of the magnetoresistance curves at larger magnetic fields is due to moving vortices.

The amplitude of the magnetoresistance oscillations becomes more pronounced in multiply connected superconductors (see, e.g., Refs. 6 and 24), which has mostly been attributed to the Little-Parks effect, ${ }^{18}$ (i.e., the periodic suppression of the superconducting critical temperature due to fluxoid quantization). ${ }^{25}$ Recently the other mechanism for the resistance oscillations in such systems have been proposed, which is based on the motion of thermally excited ${ }^{26}$ or currentinduced $^{27}$ superconducting vortices. These mechanisms are shown to better account for the amplitude and the period of the experimentally observed magnetoresistance oscillations. Our numerical simulations predict that the resistance anomalies in single connected superconducting samples can also originate from the motion of such current-induced vortices.

\section{THEORETICAL APPROACH}

We consider a superconducting strip (with thickness $d \ll$ $\xi, \lambda)$ in the presence of a uniform applied magnetic field $H$ and transport current $I$ (see Fig. 1). To understand the dynamical properties of the system we used the following time-dependent Ginzburg-Landau (TDGL) equations:

$$
\begin{gathered}
u \frac{\partial \psi}{\partial t}=(\nabla-i \mathbf{A})^{2} \psi+\left(1-|\psi|^{2}\right) \psi, \\
\frac{\partial \mathbf{A}}{\partial t}=\operatorname{Re}\left[\psi^{*}(-i \nabla-\mathbf{A}) \psi\right]-\kappa^{2} \operatorname{rot} \operatorname{rot} \mathbf{A} .
\end{gathered}
$$




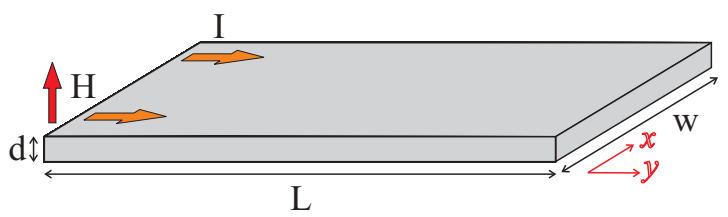

FIG. 1. (Color online) The model system: a superconducting strip (of width $w$, thickness $d \ll \lambda, \xi$, and periodically extended in the $y$ direction) in the presence of a dc current $I$ and a perpendicular magnetic field $H$.

Here distance is scaled to $\xi$, temperature is in units of the critical temperature $T_{c}$, the vector potential $\mathbf{A}$ is in units of $\Phi_{0} / 2 \pi \xi$ (where $\Phi_{0}$ is the quantum of magnetic flux), time is in units of the GL relaxation time $t_{\mathrm{GL}}=4 \pi \lambda^{2} \sigma_{n} / c^{2}\left(\sigma_{n}\right.$ is the normal state conductivity), and voltage is scaled to $\varphi_{0}=$ $\hbar / 2 e t_{\mathrm{GL}}$. The magnetic field is scaled with $H_{c 2}=\Phi_{0} / 2 \pi \xi^{2}$ and the current density with $j_{0}=\sigma_{n} \hbar / 2 e \xi t_{\mathrm{GL}}$. The coefficient $u$, which governs the relaxation of the order parameter $\psi$ (i.e., the ratio between relaxation times for the phase and the amplitude of $\psi$ ) is chosen as $u=1$, which is usually accepted in the literature (see, e.g., Ref. 28) and leads to qualitatively (and often quantitatively) good results. ${ }^{29}$ Simulations are done for a GL parameter $\kappa=4$. The above equations are solved self-consistently using the semi-implicit Crank-Nicholson algorithm $^{30}$ with periodic boundary conditions in the $y$ direction $[\psi(y)=\psi(y+L)$ and $\mathbf{A}(y)=\mathbf{A}(y+L)]$ and Neumann boundary conditions $\left[\left.(\boldsymbol{\nabla}-i \mathbf{A}) \psi\right|_{x=0, w}\right]$ in the $x$ direction. The transport current is introduced via the boundary condition for the vector potential, $\left.\operatorname{rot} \mathbf{A}\right|_{z}(x=0, w)=H \pm H_{I}$, where $H_{I}=2 \pi I / c$ is the magnetic field induced by the current $I$. Note that in Eqs. (1) and (2) the screening of the magnetic field is neglected, which is valid for extremely thin superconductors or for samples infinite in the $z$ direction.

\section{VORTEX-MOTION-INDUCED MAGNETORESISTANCE OSCILLATIONS}

We study the response of our system to increasing magnetic field for a constant external dc current, by constructing time-averaged voltage vs magnetic field $V(H)$ and voltage vs time $V(t)$ characteristics of the sample. As a representative example, we consider a superconducting strip with $w=4 \xi$, the time-averaged voltage vs magnetic field characteristics $[V(H)]$ of which are shown in Fig. 2 for different values of the current. For small values of the applied current (not shown here) the zero resistance of the sample is maintained before the system goes into the resistive state with a finite voltage jump. With increasing the applied current, a pronounced voltage peak (with amplitude larger that $30 \%$ of the normal-state voltage) is observed in the $V(H)$ curve (see solid black curve in Fig. 2). Careful analysis of the simulation results shows that this voltage signal originates from the motion of vortices across the sample (see panel 2 in Fig. 2 and discussion of Fig. 3). With further increasing $I$, both the amplitude and width of the voltage signal increases (see dashed red and dotted blue curves) due to the increased rate of the vortex nucleation and crossing process (Fig. 2, panel 6). Moreover, an extra peak appears in the $V(H)$ curve at smaller magnetic fields, which

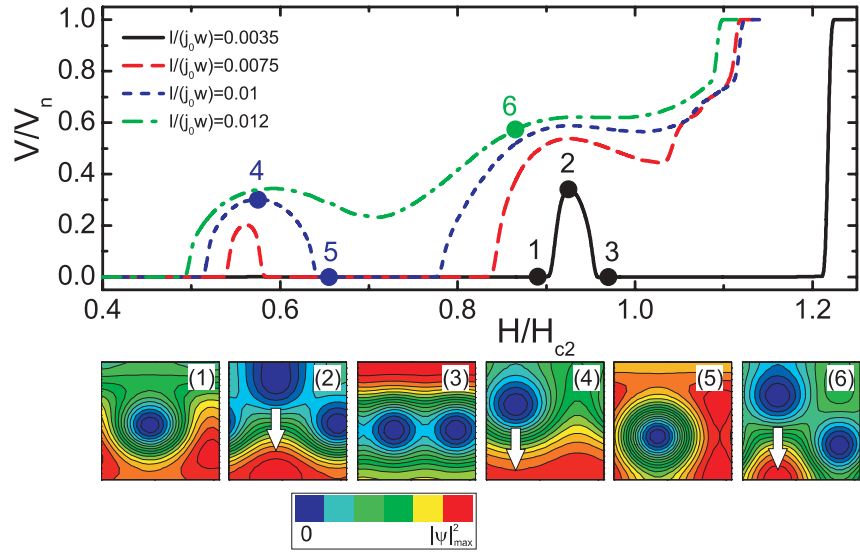

FIG. 2. (Color online) (a) Time-averaged voltage $V$ (in the units of normal state voltage $V_{n}$ ) as a function of magnetic field $H$ for different values of the applied current $I$. Panels 1-6 show snapshots of the Cooper-pair density at the field and current values indicated in the $V(H)$ curves. Arrows in panels 2, 4, and 6 indicate the direction of vortex motion. The width of the sample is $w=4 \xi$ and the size of the unit cell is $L=4 \xi$.

also results from the moving vortices (Fig. 2, panel 4). At larger currents (dash-dotted green curve), a background voltage (i.e., characteristic for a resistive state) is observed on which the magnetoresistance oscillations due to the individual entry of additional vortices superimposed (Fig. 2, panel 6).

To obtain a better insight into the process leading to the magnetoresistance oscillations, we plotted in Fig. 3(a) the time evolution of the output voltage in the sample of Fig. 2 for $I /\left(j_{0} w\right)=0.01$ and $H=0.53 H_{c 2}$, together with the snapshots of the Cooper-pair density at times indicated in the $V(t)$ curve. At this field the voltage oscillates periodically in time with a global minimum corresponding to the Meissner phase (see inset 1). With time, a vortex penetrates the sample

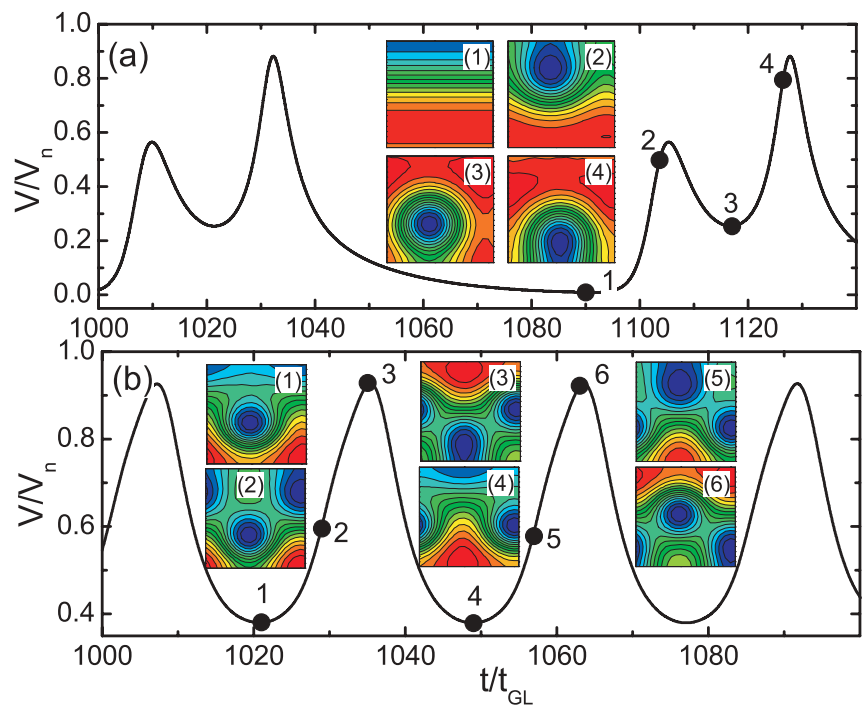

FIG. 3. (Color online) Voltage (in units of normal state voltage $\left.V_{n}\right)$ vs time characteristics of the sample in Fig. 2 at $I /\left(j_{0} w\right)=0.01$ and $H=0.53 H_{c 2}$ (a) and $I /\left(j_{0} w\right)=0.012$ and $H=0.83 H_{c 2}$ (b). Insets show snapshots of $|\psi|^{2}$ at time intervals indicated on the $V(t)$ curve. 
(inset 2), leading to a local maximum in the voltage curve (point 2). This vortex resides inside the sample for a short time interval (inset 3), at which the voltage reaches a local minimum (point 3). Later, it leaves the sample (inset 4) through a maximum in the $V(t)$ curve (point 4 ), and the system relaxes to its initial state. Immediately after, a new vortex penetrates the sample and the entire vortex entry-exit sequence repeats. Thus, the finite voltage is due to the periodic entrance of vortices. However, these vortices will be pinned inside the sample by the surface barrier when the applied field is further increased (see panel 5 in Fig. 2). For this value of the current and the magnetic field range $\left(H / H_{c 2}=0.52-0.64\right)$ the frequency of the voltage oscillations is in the range $f\left(1 / t_{\mathrm{GL}}\right)=$ $0.005-0.0155$. For a sample made of $\mathrm{Nb}$ thin film [with the normal-state resistivity $\rho_{N}=18.7 \mu \Omega \mathrm{cm}$, zero temperature coherence length $\xi(0)=10 \mathrm{~nm}$ and the penetration depth $\lambda=200 \mathrm{~nm}$ (see, e.g., Ref. 31)] the frequency of the voltage oscillations is estimated to be in the $f=2.5 \mathrm{GHz}-5.7 \mathrm{GHz}$ range.

The interplay of applied $(j)$ and screening (and circulation) currents $\left(j_{s}\right)$ turns out to be the reason that drives vortices through such alternating static and dynamic phases. The applied current $j$ enhances the supercurrent $j_{s}$ on one side of the sample (i.e., the total current is $j_{t}=j_{s}+j$ ), while suppressing $j_{s}$ on the other side $\left(j_{t}=j_{s}-j\right)$. This, in turn, directly affects the barriers for vortex entry and exit, which are indicated in Figs. 4(a) and 4(b), where we plotted the time evolution of the free energy of the system for two values of the applied magnetic field. When the total current $j_{t}$ reaches its critical value $^{32}$ the vortex nucleates at the edge of the sample in spite of the finite energy barrier $\Delta F_{p}$ [see filled circles in Fig. 4(c)]. At that field the barrier for vortex expulsion $\Delta F_{e}$ on the opposite side of the sample is strongly suppressed [see open circles in Fig. 4(c)]. As a consequence, the vortex is driven across the sample resulting in a periodic voltage versus time signal as shown in Fig. 3(a). With increasing the magnetic field, the barrier for vortex exit increases [open circles in Fig. 4(c)] and, consequently, the time interval over which the vortex is present inside the sample $\Delta t$ [see Figs. 4(a) and 4 (b)] increases. With further increasing $H$, the vortex remains trapped inside the sample and the output voltage becomes zero. Such modulation of the barrier for vortex entry and exit is observed for the transition between different vortex states (see solid black curve and compare panels 1-3 in Fig. 2).

Thus, we conclude that the magnetoresistance peaks originate from the moving vortices in the system. Note however that at higher driving current or for larger magnetic fields, it is no longer possible to stabilize the stationary vortex phase-vortices remain in motion, causing a background voltage (characteristics of a resistive state) on which the peaks for every additional vortex entry are superimposed (see dash-dotted green curve in Fig. 2). For this case, we plotted the time evolution of the output voltage together with the evolution of the superconducting condensate in Fig. 3(b). For these values of the external parameters, two rows of vortices enter the sample alternately (see the insets) leading to periodic oscillations of the $V(t)$ curve. Thus, the resistance background stems solely from continuous, dissipative vortex motion.

We would like to mention that similar resistance oscillations in singly connected mesoscopic superconductors as a
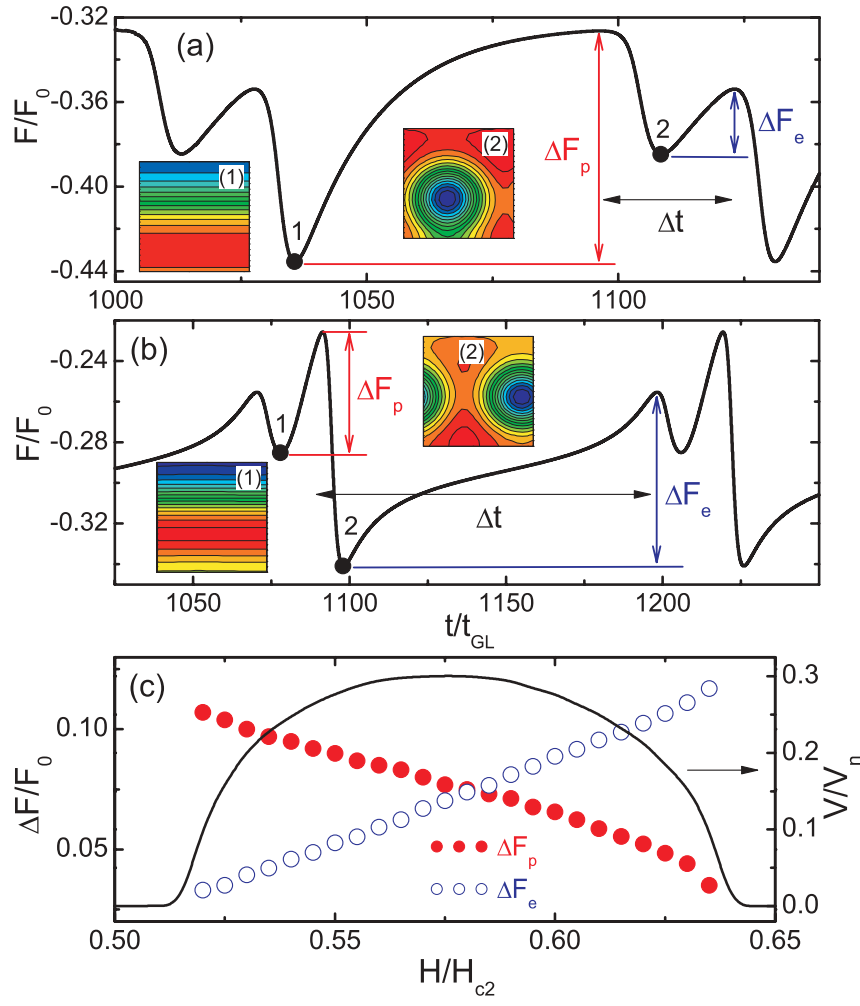

FIG. 4. (Color online) (a), (b) Time evolution of the free energy (in units of $F_{0}=H_{c}^{2} V_{0} / 8 \pi$ with $V_{0}$ being the unit cell volume) for the same sample as in Fig. 2 for $H=0.53 H_{c 2}$ (a) and $H=0.63 H_{c 2}$ (b). Vertical arrows indicate the penetration $\Delta F_{p}$ and expulsion $\Delta F_{e}$ barriers and horizontal arrow shows the time interval $\Delta t$ over which the vortex is present inside the sample. Insets show snapshots of $|\psi|^{2}$ at the times indicated on the curves. (c) Time-averaged voltage (solid curve, referred to the right axis, voltage is given in units of normal state voltage $\left.V_{n}\right), \Delta F_{p}$ (filled circles) and $\Delta F_{e}$ (open circles) as a function of the magnetic field. The applied current is $I /\left(j_{0} w\right)=0.01$.

function of external parameters have been reported in previous experiments (see, e.g., Refs. 9 and 22). Magnetoresistance oscillations observed in Ref. 22 have been associated to the formation of quantized vortices limited in size by the confinement effects. ${ }^{22}$ Fine oscillations in the experiments by Moshchalkov et al. in mesoscopic superconductors as a function of temperature have been related to quantum interference processes, which results in the formation of superconducting but non-current-carrying states in the system. Our numerical simulations support the predictions of Anderson and Dayem ${ }^{23}$ that the voltage oscillations in finite width superconducting strips originate from the alternating static and dynamic regimes in the dynamics of superconducting vortices.

In what follows, we study the effect of the strip width $w$ on its transport properties. Figure 5 shows the time-averaged voltage as a function of the flux (calculated over the unit cell area) for different widths of the sample $w$ and for the same value of the applied current density. As one can see from this figure, the size of the first voltage peak (corresponding to one row of moving vortices, see inset 1) decreases with increasing $w$ (compare solid black and dashed red curves) and eventually disappears starting from $w>8 \xi$. The entry of the first row of vortices will not bring any resistance anomalies for 


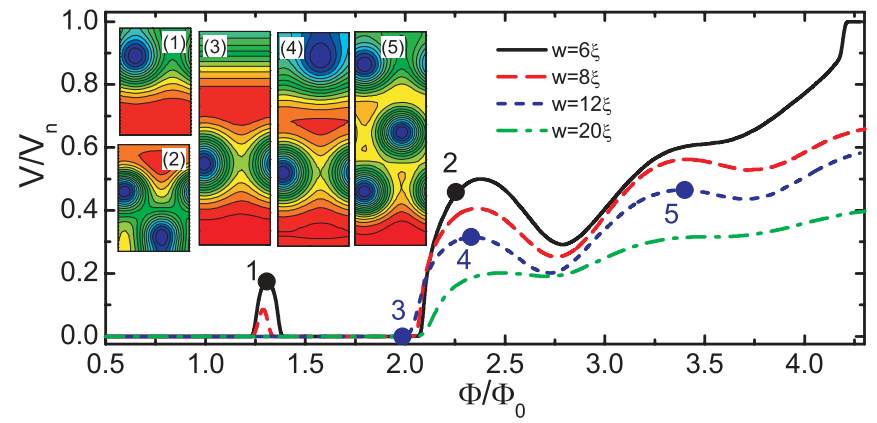

FIG. 5. (Color online) Time-averaged voltage (in units of normal state voltage $V_{n}$ ) as a function of flux $\Phi$ (calculated over the unit cell area $S=w \times L$ ) for different width of the sample. The applied current density is $j=0.04 j_{0}$.

wider samples (dashed blue curve), since the vortices will be completely pinned inside the sample (Fig. 5, inset 3). This is due to the bulk pinning, which comes into play with increasing $w$. Note the displacement of the vortices due to the external current (inset 3). One can observe more resistance peaks for wider samples due to the formation of extra vortex rows (see insets 4 and 5). However, the amplitude of those extra voltage peaks will be suppressed and they are always superimposed on the resistance background (see dotted blue and dash-dotted green curves).

\section{EFFECT OF DISORDER}

It is well known that the response of a superconductor to external magnetic field is strongly affected by the disorder in the system and by edge imperfections, ${ }^{33}$ which is inevitable in real superconductors. For example, the critical field for the penetration of vortices in type-II superconductors is strongly reduced by introducing edge defects (see, e.g., Ref. 32), resulting in qualitative changes in the magnetization curves of the system. ${ }^{32}$ The disorder also results in multiple reentrant transitions between the superconducting and normal states by increasing the applied current (see, e.g., Ref. 17). Defects on the mesoscopic scale can also result in magnetoresistance oscillations similar to Little-Parks oscillations due to the formation of effective multiconnected (ringlike) structures.

In this section we study the effect of edge imperfections and inhomogeneities on the magnetic and dissipative properties of superconducting strips. We include disorder as nonmagnetic inclusions of size comparable to the superconducting coherence length $\xi$ with reduced transition temperature. These weakly superconducting regions are described by spatially changing the coefficient $\alpha$ in the Ginzburg-Landau free energy expression. ${ }^{34}$ This modifies the last term of Eq. (1) as $\left(v-|\psi|^{2}\right) \psi,{ }^{35}$ where the spatially dependent parameter $v$ is given by $v<0$ inside the defect and $v=1$ otherwise and characterizes the attractive strength of the defect. As a typical example, we consider a superconducting strip with width $w=5 \xi$ and length $L=15 \xi$ with eight randomly generated defects of radius $r=1 \xi$ and pinning strength $\nu=0.5$ (the location of the defects are indicated by white circles in panels 2-4 of Fig. 6). We consider the case when the defects are present both at the sample surface and at the interior of the sample. We would like to mention that, although the
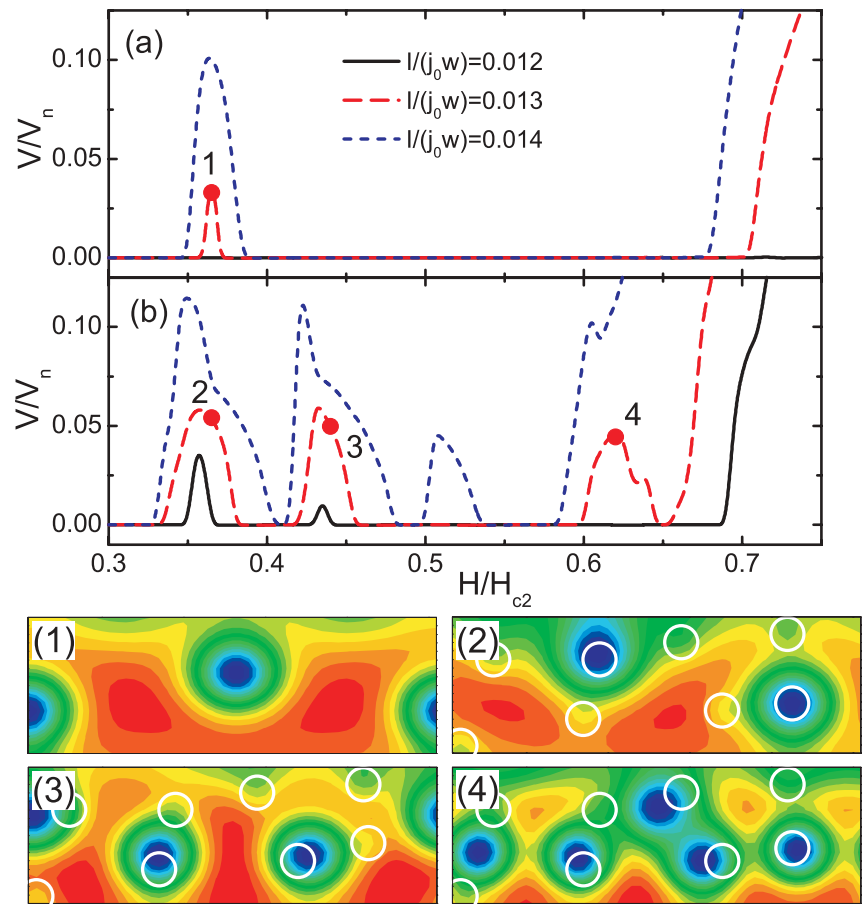

FIG. 6. (Color online) Time-averaged voltage $V$ (in units of normal state voltage $V_{n}$ ) as a function of magnetic field $H$ for the sample with $w=5 \xi$ and $L=15 \xi$ without (a) and with eight randomly generated defects of radius $r=0.5 \xi$ (b) inside of which the superconducting transition temperature is suppressed. Results are shown for three different values of the applied current. Panels 1-4 show snapshots of $|\psi|^{2}$ for current and magnetic field values indicated on the $V(t)$ curves. White circles in panels 2-4 indicate the positions of the defects.

anisotropy parameter $v$ changes stepwise at the defect interface, the order parameter changes smoothly at the interface due to the proximity effect. ${ }^{36}$ Figure 6 shows the $V(H)$ curves of the sample without (a) and with (b) the defects for three different values of the applied current. The presence of the defects changes the voltage curve of the sample considerably: (i) voltage oscillations are observed at lower values of the applied current [compare solid black curves in Figs. 6(a) and 6(b)] and magnetic field (dashed red curves); (ii) voltage peaks become wider and their amplitude increases when defects are present [compare dashed blue curves in Figs. 6(a) and 6(b)]; (iii) more voltage peaks are observed in the defective sample before the monotonic background in the $V(H)$ curve is reached (for the given parameter set we observed three voltage peaks); and (iv) irregularities are observed in the voltage signal [dotted blue curve in Fig. 6(b)].

It has been shown in previous theoretical studies (see, e.g., Ref. 32) that the modulation instability of the superconducting order parameter occurs when the kinematic momentum (or supervelocity) of the condensate reaches a threshold value at the edge of the superconductor. This results in the formation of a chain of vortices in the system (see panel 2 in Fig. 8). However, this condition is reached near the surface defects (due to crowding of the supercurrents near the defects) ${ }^{37}$ at magnetic field values smaller than the one for the uniform sample. As a consequence, vortices penetrate the sample through the surface 

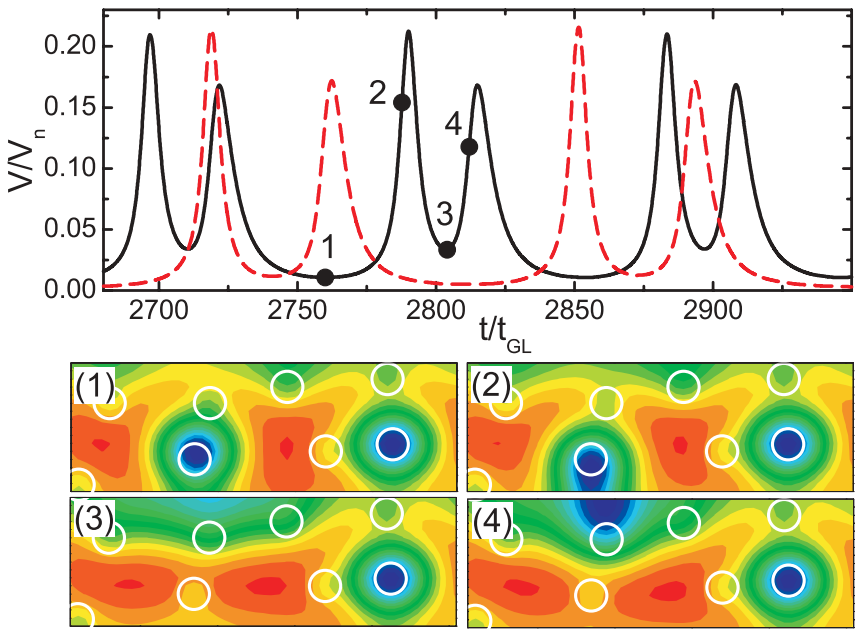

FIG. 7. (Color online) Voltage vs time characteristics of the sample of Fig. 6 without (dashed red curve) and with (solid black curve) defects at $I /\left(j_{0} w\right)=0.013$ and $H=0.365 H_{c 2}$. Panels $1-4$ show snapshots of $|\psi|^{2}$ at time intervals indicated on the $V(t)$ curve.

defects (see panel 2 in Fig. 6) reducing the critical field for the voltage oscillations. The expulsion barrier of the vortices is also affected by the defects located near the other edge of the sample, which results in a widening of the voltage peaks to higher magnetic fields. As we will show in Fig. 7, for some location of the defects an easy channel is formed for vortex crossing across the sample. As the time-averaged voltage is directly proportional to the net rate with which vortices cross the strip, the latter explains the increased voltage peaks in the presence of defects [Fig. 6(b)]. Once vortices are trapped inside the sample, we need to increase the magnetic field further to reach the condition for the penetration of extra vortices. The defects reduce the penetration barrier and the above condition is reached earlier. As a consequence, we observe more voltage peaks in the $V(H)$ curve. We relate the irregularities observed in the magnetoresistance curves [dotted blue curve in Fig. 6(b)] to the randomness of the defects (see white circles in panels 2-4 of Fig. 6). These results resemble the nonperiodic behavior of the resistance oscillations usually observed in experiments. ${ }^{15,16}$

To see the dynamics of vortices in the presence of defects we plotted in Fig. 7 the voltage vs time characteristics of the sample in Fig. 6 together with snapshots of the spatial variation of the order parameter at times indicated on the $V(H)$ curve. For the given values of the external parameters $\left[I /\left(j_{0} w\right)=0.013\right.$ and $\left.H=0.365 H_{c 2}\right]$, the global minimum in the voltage curve corresponds to two vortices trapped inside the sample (Fig. 7, panel 1). Both of the vortices are pinned by the defects. With time going on the vortex close to the lower boundary leaves the sample (Fig. 7, panel 2), resulting in a maximum in the $V(t)$ curve (point 2). Thus, the next minimum in the voltage curve corresponds to the state with a single vortex pinned in the defect (Fig. 7, panel 3). Later, another vortex enters the sample through the defect that is close to the surface (panel 4) and it will be pinned in the same defect as before (panel 1). This periodic process results in periodic oscillations in the voltage signal across the sample. Note that one vortex is always immobile due to the pinning and the other vortices move through an "easy" channel formed by the other defects, whereas in a defect free sample vortices oscillate alternately [see Fig. 3(b)]. Formation of this channel results in a considerable decrease of the period of voltage oscillations (compare solid and dashed curves in Fig. 7) and explains the increase of the voltage peaks in the time-averaged voltage vs magnetic field characteristics of the system [see Fig. 6(b)].

Thus, the presence of defects results in qualitative changes in the magnetoresistance curves of superconducting strips. This is because of the influence of the defects to both static (panel 4 in Fig. 6) and dynamic (panels 1-4 in Fig. 7) properties of vortices. The resistance oscillations in a defective sample is also due to the moving vortices.

\section{EFFECT OF SUPERCONDUCTING CURRENT CONTACTS}

In most of the experiments, including the pioneering works of Park and Mochel ${ }^{22}$ and the famous work of Anderson and Dayem, ${ }^{23}$ a bridge geometry is used, where a superconducting strip is connected to large superconducting leads. As was shown in numerical simulations by Vodolazov et al. ${ }^{38}$ such connecting regions can play a role of a "weak" point for the nucleation of phase-slip centers at sufficiently large applied currents. Generation of a local charge imbalance around such phase-slip centers can results in strong nonlocal effects, manifesting itself as, for example, local enhancement of the resistance above the normal state value. Such pronounced voltage peaks near the superconducting transition temperature has been reported previously in quasi-one-dimensional structures with attached extra contacts (see, e.g., Ref. 7).

In what follows, we study the effect of such superconducting contacts to our findings. For the system shown in the inset of Fig. 8, we solved the following TDGL equations: ${ }^{39}\left(\frac{\partial}{\partial t}+i \varphi\right) \psi=(\nabla-i \mathbf{A})^{2} \psi+\psi-$ $|\psi|^{2} \psi$ and $\frac{\partial \mathbf{A}}{\partial t}=\operatorname{Re}\left[\psi^{*}(-i \nabla-\mathbf{A}) \psi\right]-\kappa^{2} \operatorname{rot} \operatorname{rot} \mathbf{A}$, where $\varphi(x, y)=j x$ is the electric potential and $j$ denotes the applied current density. Assuming that the applied magnetic field $H$ is always perpendicular to the sample, we neglect the formation of curved vortices in the $z$ direction and carry out the simulations on a two-dimensional (2D) space grid. To characterize the vortex dynamics and phase transitions in the system under applied current and magnetic field, we used the time average of $\partial|\psi| / \partial t \equiv|\psi|_{t}$.

Figure 8 shows the magnetic field dependence of $|\psi|_{t}$ for the sample with $L=32 \xi$ and $W=4 \xi$ for different values of the applied current density $j$, together with the snapshots of $|\psi|$ for current and magnetic field values indicated on the $|\psi|_{t}(H)$ curves. For small values of the applied current (black circles) the system is in the Meissner state until a critical magnetic field is reached (see Fig. 8, panel 1), starting from which a row of vortices penetrates the sample (Fig. 8, panel 2). These vortices are pinned inside the sample and no dissipation is observed (point 2). With further increasing $H$ vortices start moving (Fig. 8, panel 3) and form easy-flow channels across the sample. The latter is clearly seen in the trajectories of the vortices plotted in Fig. 8, panel 3'. At larger field superconductivity is completely suppressed and the system transits into the normal state (Fig 8, panel 4). 

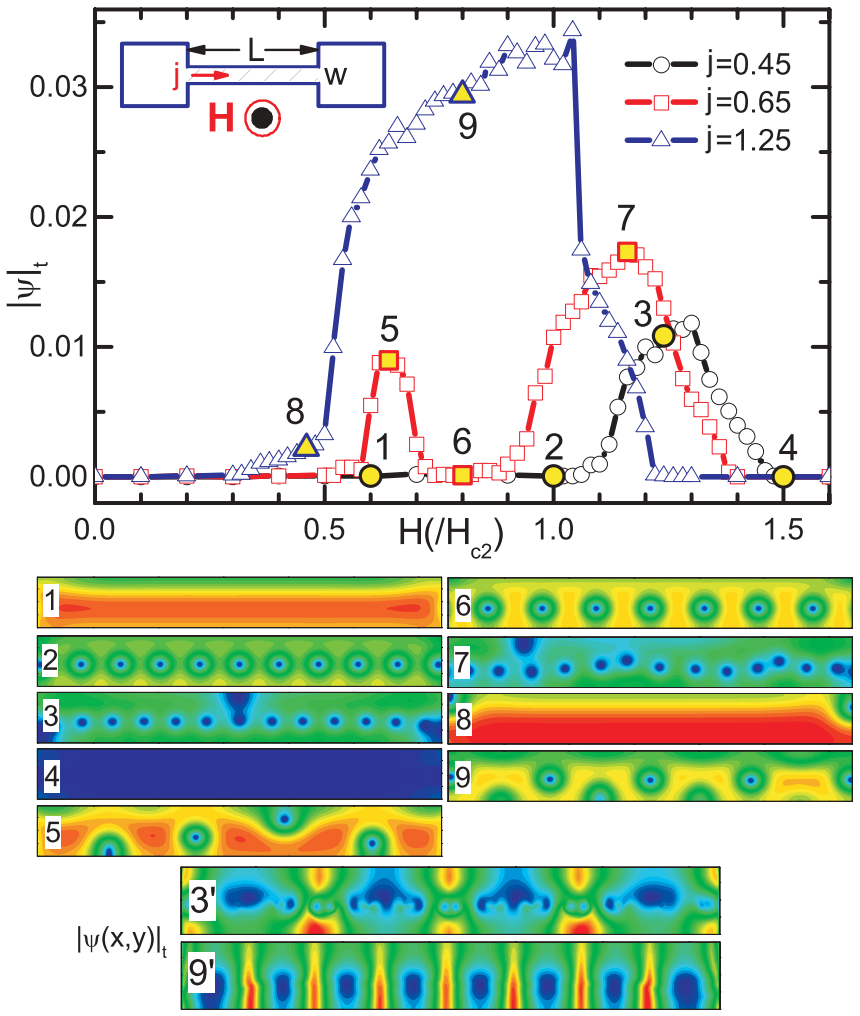

FIG. 8. (Color online) The average $|\psi|_{t}$ vs applied magnetic field for the sample with $L=32 \xi$ and $W=4 \xi$ for different current densities $j$. The inset shows the system setup used for the simulations. Panels 1-9 show snapshots of $|\psi|$ for the current and field values indicated in the main panel. Panels $3^{\prime}$ and $9^{\prime}$ show contour plots of the time average of $|\psi|_{t}$ from which the trajectories of the moving vortices can be clearly identified. The GL parameter is $\kappa=2.2$.

With increasing applied current, the $|\psi|{ }_{t}(H)$ curve shows a distinct double peak behavior (red squares). The peaks together with the region $|\psi|_{t}(H)=0$ between them are due to the alternating phase transitions between the vortex flow and pinning (normal) states that are tuned by the external field, as we have discussed above. In the flux flow regime (point 5) alternating entrance of vortices is observed (panel 5), which is due to repulsive interactions between vortices in type-II superconductors. In this particular case the number of vortices inside the sample varies between three and four. With further increasing $H$, all seven vortices will be pinned inside the sample (Fig. 8, panel 6) and no dissipation is observed (point 6). At larger magnetic fields, irregular motion of vortices can be observed (Fig. 8, panel 7). Thus, regardless of the sample geometry (i.e., infinitely long strips or short samples connected to bulk superconductors) resistance oscillations as a function of magnetic field can be observed due to the moving vortices.

However, the effect of the superconducting leads becomes more obvious at larger current values, at which the critical magnetic field for the dissipation onset becomes weaker, as shown by the blue triangles in the main panel of Fig. 8 for $j=1.25$. In this case, just before the transition to the highly resistive state (point 8 ), although there are no vortices in the sample (Fig. 8, panel 8), vortex motion near the electrodes has a clear influence and leads to a nontrivial $|\psi|_{t}(H)$ response. With further increasing $H$, the vortices enter and leave the
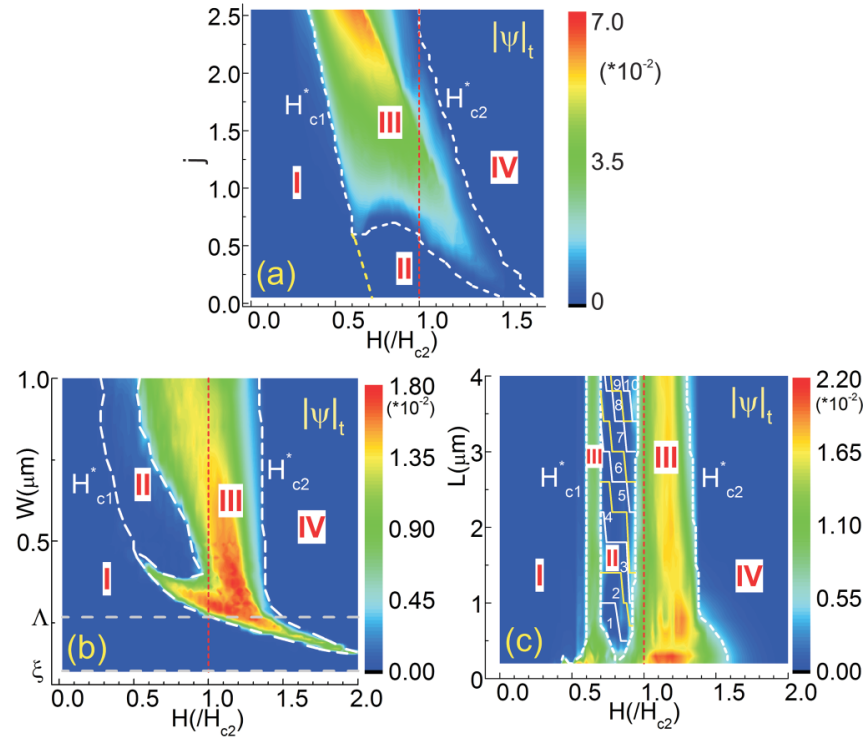

FIG. 9. (Color online) Phase diagrams: $|\psi|_{t}$ versus the magnetic field $H$ and (a) the current density $j$ for $L=32 \xi$ and $W=4 \xi$; (b) the width $W$ for fixed length $L=32 \xi$ at $j=0.65$; (c) the length $L$ for fixed width $W=4 \xi$ at $j=0.65$. Regions I-IV indicate four different phases (see text for the description of different phases) separated by the white dashed lines and the Arabic numbers in (c) indicate the number of vortices pinned in the sample. The vertical red/dotted lines highlight the upper critical magnetic field $H_{c 2} . H_{c 1}^{*}$ and $H_{c 2}^{*}$ shows the transitions from the Meissner state to the vortex state and from the vortex state to the normal state, respectively.

sample row by row with fixed intervortex spacing (see Fig. 8, panels 9 and $9^{\prime}$ ). Thus, a Bragg glass phase of vortex motion can also be observed in our system.

Our findings are summarized in Fig. 9, where we constructed three phase diagrams showing the effect of the applied current and sample dimensions to the transitions between different superconducting phases with intrinsic properties. These phases are: I, the Meissner state; II, vortex pinning state; III, moving vortex phase; and IV, normal state. As seen from Fig. 9(a), the peak response of $|\psi|_{t}(H)$ (i.e., the reentrant behavior of the phase III as a function of $H$ ) is observed only for the relatively low magnetic fields and only in a limited current region, which can be attributed to the matching between the fields and the sample size. At the proper field region, the number of vortices may not be exactly an integer for the matching effect, but the vortex density in the sample will ensure a favorable vortex spacing for the proper vortex-vortex interaction, which results in a stable vortex flow state. With increasing the applied current the two separate vortex flow regions are gradually merged into a single one. As expected, the critical magnetic fields for the transition between the Meissner state and the vortex state $H_{c 1}^{*}$ and between the superconducting state and the normal state $H_{c 2}^{*}$ decrease gradually with increasing applied current.

As we have shown in Fig. 5, novel transport properties can be obtained by increasing the width of the sample. Figure 9(b) shows the detailed analysis of the effect of the sample width on the transitions between different superconducting phases. For narrow samples, the system transits to the phases I, III, and IV with increasing the magnetic field. Clear alternating phase 
transitions between phase II (pinned vortices) and phase III (moving vortices) occur only for intermediate values of the sample width. For larger transverse dimensions, the system crosses all four phases consecutively with increasing field. However, the level of dissipation is not constant for fixed width of the sample in the flux regime, as was discussed in Fig. 5. The critical magnetic fields of the sample are also affected by the width of the sample: $H_{c 1}^{*}$ decreases gradually with $W$ at small sample sizes, followed by a sharp decrease of $H_{c 1}^{*}$ for wider samples. Whereas, the second critical field $H_{c 2}^{*}$ becomes almost independent of $W$ after a certain critical width. However, the magnetic field values for the alternating phase transitions are almost independent on the length of the sample $L$, as shown in Fig. 9(c). This is in spite of the fact that the number of vortices present in the system increases with $L$ [see Arabic numerals in Fig. 9(c)]. Note that the broadening of the region III for smaller $L$ is due to the effect of the superconducting leads (see the discussions of panel 8 of Fig. 8).

\section{CONCLUSION}

In summary, we have readdressed the problem of magnetoresistance oscillations in superconducting samples with transverse dimensions larger than the superconducting coherence length. We found that such oscillations result from the nucleation, motion, and stabilization of superconducting vortices due to the interplay of the driving current and the persistent currents in the system. Our findings confirm the predictions of Anderson and Dayem ${ }^{23}$ about the vortexassisted resistance oscillations in narrow superconducting strips. Continuously moving vortices also contribute to the background voltage signal on which the voltage peaks due to the formation of extra rows of vortices are superimposed. Magnetoresistance oscillations are sensitive to the external parameters (e.g., the applied current), as well as to the transverse dimensions of the sample because of the bulk pinning, which becomes important for wider samples. Periodic motion of superconducting vortices is strongly affected by the disorder in the system, which results in qualitative changes in the magnetoresistance curves of the system. However, even in a defective sample resistance peaks are observed due to the moving vortices.

\section{ACKNOWLEDGMENTS}

This work was supported by the Flemish Science Foundation (FWO-Vl) and the ESF-NES program. G.R.B. acknowledges support from FWO-Vl. B.Y.Z. acknowledges the support from the MOST 973 Projects No. 2011CBA00110 and No. 2009CB930803, and the National Natural Science Foundation of China. V.V.M. acknowledges support from the Methusalem Funding by the Flemish Government. *francois.peeters@ua.ac.be

${ }^{1}$ J. M. Blatt and C. J. Thompson, Phys. Rev. Lett. 10, 332 (1963).

${ }^{2}$ M. Strongin, R. S. Thompson, O. F. Kammerer, and J. E. Crow, Phys. Rev. B 1, 1078 (1970).

${ }^{3}$ A. Perali, A. Bianconi, A. Lanzara, and N. L. Saini, Solid State Commun. 100, 181 (1996).

${ }^{4}$ J. E. Han and V. H. Crespi, Phys. Rev. B 69, 214526 (2004).

${ }^{5}$ A. A. Shanenko, M. D. Croitoru, M. Zgirski, F. M. Peeters, and K. Arutyunov, Phys. Rev. B 74, 052502 (2006); A. A. Shanenko, M. D. Croitoru, and F. M. Peeters, ibid. 75, 014519 (2007); 78, 054505 (2008); A. A. Shanenko, M. D. Croitoru, R. G. Mints, and F. M. Peeters, Phys. Rev. Lett. 99, 067007 (2007).

${ }^{6}$ V. V. Moshchalkov, L. Gielen, C. Stunk, R. Jonckheere, X. Qiu, C. Van Haesendonck, and Y. Bruynseraede, Nature (London) 373, 319 (1995).

${ }^{7}$ C. Strunk, V. Bruyndoncx, C. Van Haesendonck, V. V. Moshchalkov, Y. Bruynseraede, C.-J. Chien, B. Burk, and V. Chandrasekhar, Phys. Rev. B 57, 10854 (1998).

${ }^{8}$ V. V. Moshchalkov, L. Gielen, G. Neuttiens, C. Van Haesendonck, and Y. Bruynseraede, Phys. Rev. B 49, 15412 (1994); C. Strunk, V. Bruyndoncx, C. Van Haesendonck, V. V. Moshchalkov, Y. Bruynseraede, B. Burk, C.-J. Chien, and V. Chandrasekhar, ibid. 53, 11332 (1996).

${ }^{9}$ V. V. Moshchalkov, L. Gielen, M. Dhalldé, C. Van Haesendonck, and Y. Bruynseraede, Physica B 194, 1617 (1994).

${ }^{10}$ B. Burk, C.-J. Chien, V. Chandrasekhar, C. Strunk, V. Bruyndoncx, C. Van Haesendonck, V. V. Moshchalkov, and Y. Bruynseraede, Superlattices Microstruct. 20, 575 (1996).
${ }^{11}$ A. A. Shanenko, M. D. Croitoru, A. Vagov, and F. M. Peeters, Phys. Rev. B 82, 104524 (2010).

${ }^{12}$ J. S. Langer and V. Abegaokar, Phys. Rev. 164, 498 (1967); D. E. McCumber and B. I. Halperin, Phys. Rev. B 1, 1054 (1970).

${ }^{13}$ N. Giordano, Phys. Rev. Lett. 61, 2137 (1988); A. D. Zaikin, D. S. Golubev, A. van Otterlo, and G. T. Zimanyi, ibid. 78, 1552 (1997); D. S. Golubev and A. D. Zaikin, Phys. Rev. B 64, 014504 (2001).

${ }^{14}$ A. Johansson, G. Sambandamurthy, D. Shahar, N. Jacobson, and R. Tenne, Phys. Rev. Lett. 95, 116805 (2005).

${ }^{15}$ U. Patel, S. Avci, Z. L. Xiao, J. Hua, S. H. Yu, Y. Ito, R. Divan, L. E. Ocola, C. Zheng, H. Claus, J. Hiller, U. Welp, D. J. Miller, and W. K. Kwok, Appl. Phys. Lett. 91, 162508 (2007).

${ }^{16}$ J. Wang, Xu-Cun Ma, Li Lu, Ai-Zi Jin, Chang-Zhi Gu, X. C. Xie, Jin-Feng Jia, Xi Chen, and Qi-Kun Xue, Appl. Phys. Lett. 92, 233119 (2008)

${ }^{17}$ B. Spivak and F. Zhou, Phys. Rev. Lett. 74, 2800 (1995).

${ }^{18}$ W. A. Little and R. D. Parks, Phys. Rev. Lett. 9, 9 (1962); Phys. Rev. 133, A97 (1964).

${ }^{19}$ U. Patel, Z. L. Xiao, A. Gurevich, S. Avci, J. Hua, R. Divan, U. Welp, and W. K. Kwok, Phys. Rev. B 80, 012504 (2009); A. V. Herzog, P. Xiong, and R. C. Dynes, ibid. 58, 14199 (1998).

${ }^{20}$ M. Vinokur, T. I. Baturina, M. V. Fistul, A. Yu. Mironov, M. R. Baklanov, and C. Strunk, Nature (London) 452, 613 (2008).

${ }^{21}$ T. I. Baturina, V. M. Vinokur, A. Yu. Mironov, N. M. Chtchelkatchev, D. A. Nasimov, and A. V. Latyshev, Europhys. Lett. 93, 47002 (2011).

${ }^{22}$ R. D. Parks and J. M. Mochel, Phys. Rev. Lett. 11, 354 (1963); Rev. Mod. Phys. 36, 284 (1964); R. D. Parks, J. M. Mochel, and L. V. Surgent, Jr., Phys. Rev. Lett. 13, 331 (1964). 
${ }^{23}$ P. W. Anderson and A. H. Dayem, Phys. Rev. Lett. 13, 195 (1964).

${ }^{24}$ R. P. Groff and R. D. Parks, Phys. Rev. 176, 567 (1968); M. Morelle, D. S. Golubovic, and V. V. Moshchalkov, Phys. Rev. B 70, 144528 (2004); G. R. Berdiyorov, S. H. Yu, Z. L. Xiao, F. M. Peeters, J. Hua, A. Imre, and W. K. Kwok, ibid. 80, 064511 (2009).

${ }^{25}$ F. London, Phys. Rev. 74, 562 (1948); B. S. Deaver Jr. and W. M. Fairbank, Phys. Rev. Lett. 7, 43 (1961); R. Doll and M. Näbauer, ibid. 7, 51 (1961).

${ }^{26}$ I. Sochnikov, A. Shaulov, Y. Yeshurun, G. Logvenov, and I. Bozovic, Nature Nanotech. 5, 516 (2010).

${ }^{27}$ G. R. Berdiyorov, M. V. Milošević, M. L. Latimer, Z. L. Xiao, W. K. Kwok, and F. M. Peeters, Phys. Rev. Lett. 109, 057004 (2012).

${ }^{28}$ V. V. Baranov, A. G. Balanov, and V. V. Kabanov, Phys. Rev. B 84, 094527 (2011).

${ }^{29}$ B. I. Ivlev, N. B. Kopnin, and L. A. Maslova, Zh. Eksp. Teor. Fiz. 78, 1963 (1980) [Sov. Phys. JETP 51, 986 (1980)].

${ }^{30}$ T. Winiecki and C. S. Adams, J. Comput. Phys. 179, 127 (2002); D. Yu. Vodolazov and F. M. Peeters, Phys. Rev. B 76, 014521 (2007).

${ }^{31}$ A. I. Gubin, K. S. Il'in, S. A. Vitusevich, M. Siegel, and N. Klein, Phys. Rev. B 72, 064503 (2005).
${ }^{32}$ D. Y. Vodolazov, I. L. Maksimov, and E. H. Brandt, Physica C 384, 211 (2003).

${ }^{33}$ P. G. de Gennes, Superconductivity of Metals and Alloys (AddisonWesley, Redwood City, California, 1989).

${ }^{34} \mathrm{M}$. Tinkham, Introduction to Superconductivity, 2nd ed. (McGrawHill, New York, 1996).

${ }^{35}$ R. Kato, Y. Enomoto, and S. Maekawa, Physica C 227, 387 (1994).

${ }^{36}$ C.-Yu. Liu, G. R. Berdiyorov, and M. V. Milošević, Phys. Rev. B 83, 104524 (2011); G. R. Berdiyorov, A. R. de C. Romaguera, M. V. Milošević, M. M. Doria, L. Covaci, and F. M. Peeters, Eur. Phys. J. B 85, 130 (2012).

${ }^{37}$ J. R. Clem and K. K. Berggren, Phys. Rev. B 84, 174510 (2011); J. R. Clem, Y. Mawatari, G. R. Berdiyorov, and F. M. Peeters, ibid. 85, 144511 (2012).

${ }^{38}$ D. Y. Vodolazov, F. M. Peeters, M. Morelle, and V. V. Moshchalkov, Phys. Rev. B 71, 184502 (2005).

${ }^{39}$ M. Machida and H. Kaburaki, Phys. Rev. Lett. 71, 3206 (1993); R. Kato, Y. Enomoto, and S. Maekawa, Phys. Rev. B 47, 8016 (1993); Q. Du, Contemp. Math. 329, 105 (2003); W. D. Gropp, H. G. Kaper, G. K. Leaf, D. M. Levine, M. Palumbo, and V. M. Vinokur, J. Comput. Phys. 123, 254 (1996). 